\title{
INTEGRATING STEEPEST ASCENT FOR THE TAGUCHI EXPERIMENT: A SIMULATION STUDY
}

\author{
M. Arbi Hadiyat ${ }^{*}$, Rahman Dwi Wahyudi ${ }^{1}$ \\ ${ }^{1}$ Industrial Engineering Department, Faculty of Engineering, University of Surabaya (Ubaya), \\ Surabaya 60293, Indonesia
}

(Received: February 2013 / Revised: June 2013 / Accepted: July 2013)

\begin{abstract}
Many previous researches conveyed the superiority of Steepest Ascent (SA) method to find the optimal area in Response Surface Methodology (RSM) by shifting the experiment factor level. By using this method, Design of Experiment (DoE) is enabled to shift the factor level gradually in the right track, so that the global optimum can be reached. However, the response variable that is commonly optimized by using RSM cannot fulfill the classical statistics assumption of surface regression model. Taguchi's orthogonal array, as alternative of RSM, gives loose statistics assumptions in performing the analysis. However, Taguchi's orthogonal array has not yet been supported to shift the factor level to an optimum direction. Adopting the procedures of RSM in finding the optimal level combination using SA, integrating SA method in the Taguchi experiment is proposed in this paper. This procedure is applied into a simulated response surface. Then, the performance of this procedure is evaluated based on its direction to reach the optimum solution. The simulation data representing the real case is generated for two factors. Then, the proposed procedure is applied. The result of this simulation study shows that the integrated SA method in the Taguchi experiment successfully found the factor level combination that yields optimum response even though it is not as close as possible as the RSM results.
\end{abstract}

Keywords: Optimum response; Regression model; Response Surface Methodology; Steepest ascent; Taguchi

\section{INTRODUCTION}

Taguchi has introduced offline quality control when quality problems can't be solved only by using classical online quality control. Presently, the role of offline quality control has been increasingly important in the quality improvement process. In the quality improvement experiment, the parameter of designing a product (such as identify factors, blocks, levels, DoE, and so on) becomes the initial step causing unconformity of response during the manufacturing process. In this situation, an experimental design method has completed this offline quality control to optimize the industrial tools setting and to obtain the robust parameter design.

Fisher (1890-1962), in Box (1980), Box and Draper (1987), and Stanley (1966), the first originators, introduced the DoE through his book "The Arrangement of Field Experimentress" (1926). They explained that DoE as a method for analyzing experimental result in agricultural field. In these results, the classical DoE such as Completely Randomized Design (CRD), Randomized Block Design (RBD) and also the well-known Factorial Design were mostly applied to help the researcher in investigating single or multifactor effects. 